\title{
Studying the Racial and Ethnicity Effects on Breastfeeding for Adolescent Mothers: Healthcare Policy Implications
}

\author{
Javis Knott ${ }^{1 *}$, Denitra C. Mitchell ${ }^{2}$ \\ ${ }^{1}$ School of Public Health, Jackson State University, 350 W. Woodrow Wilson Drive, Suite 320, Jackson, MS 39213-7681, USA. \\ ${ }^{2}$ Administrator/Government Contract Specialist
}

\begin{abstract}
Article Details
Article Type: Mini Review

Received date: $23^{\text {rd }}$ September, 2019

Accepted date: $22^{\text {nd }}$ October, 2019

Published date: $25^{\text {th }}$ October, 2019
\end{abstract}

"Corresponding Author: Javis Knott, School of Public Health, Jackson State University, 350 W. Woodrow Wilson Drive, Suite 320, Jackson, MS 39213-7681, USA. E-mail: Javis.m.knott@jsums.edu

Citation: Knott J, Mitchell DC (2019) Studying the Racial and Ethnicity Effects on Breastfeeding for Adolescent Mothers: Healthcare Policy Implications. J Pub Health Issue Pract 3: 147. doi: https://doi.org/10.33790/jphip1100147.

Copyright: $\left({ }_{0} 2019\right.$, This is an open-access article distributed under the terms of the Creative Commons Attribution License 4.0, which permits unrestricted use, distribution, and reproduction in any medium, provided the original author and source are credited.

\begin{abstract}
According to the American Association of Pediatrics on the racial and ethnic differences of breastfeeding adolescent mother's show that a total of 696 Latino, African and Caucasian adolescent mothers under the age of 18 were studied. The study highlighted the factors that influence adolescent mothers to breastfeed. These results were reported that majority of the Mexican American mothers were likely to breastfeed. The decision to breastfeed stated 55\% of MexicanAmerican, $45 \%$ of Caucasian, and $15 \%$ of African-American adolescent mothers did in fact breastfeed. With the exception of perceived benefits of breastfeeding and exposure to educational materials, most factors associated with breastfeeding differed by race/ethnicity.
\end{abstract}

Among Mexican-Americans, important factors included feeding advice. The feeding preference of a partner or mother and feeding decisions were made in early pregnancy. Among African-Americans, important factors included living with a partner, having a mother who breastfed. The feeding preference of a partner or health care provider and low family support was a factor. Among Caucasians, health care providers' feeding preference having two or more breastfeeding role models not being enrolled in Women, Infants, and Children's Supplemental Nutrition Program having relied on infant-feeding advice was associated with the decision to breastfeed.

The purpose of this study is to focus on racial and ethnic differences when it comes to breastfeeding. As adolescent mothers, women have to decide what best is for their children. Therefore, it is important to have information available to best help them decide despite cultural biases. Specific members of the adolescent's support systems that were identified in this study as important to her feeding decision. Medical and Social Groups must focus on the benefits of breastfeeding and being able to address concerns and refute fallacies common to young mothers specific to each racial/ethnic group. Also identified is the need to incorporate plenty of educational reading materials. Finally, a special effort must be made to overcome biases in the health care system by training health care professionals to encourage breastfeeding among all adolescent mothers, including African-Americans. Remain adhere of involvements could target both the adolescent mother and her support system.

\section{Introduction}

Women have been breastfeeding since the existence of man. It has

always been a natural thing for a mother breastfeed her new born baby.However, with the invention of formula, breastfeeding has become so taboo. Much of the literature in the past generalized women in regards to breastfeeding. The literature also did not focus much on breastfeeding of African American women. There seems to have been a shift in society from breast feeding as a way for a mother to nourish her baby and more focus on the female anatomy. The breast has a vital role such as sustain milk for an infant, as well as stimulating a sexual sense. The thought, concept and culture of the black woman's autonomy have undergone the most scrutiny because of ignorance. Breastfeeding started as a lucrative business, but not for African Americans, who came to this country as slaves.

In Africa,the women exclusivelybreastfed their children well beyond the first year of life.After being forced from their home and sold into bondage they were forced to wet nurse the Plantation owner's child.Wet nursing is defined as a lactating woman who breastfeeds a child that is not her own. There may be many factors that could contribute to the possibility that breastfeeding is no longer viewed positively in the African American community. As defined by The Journal of Perinatal Education at the National Center for Biotechnology Information, U.S. National Library of Medicine, Spring 2009. Many continued as wet nurses for white families even after slavery.This historical legacy of breastfeeding as unpaid or low paid labor may have had an adverse effect on black women's attitudes towards breastfeeding.

Perhaps the reason for this thinking is rooted in slavery and poverty. During slavery, African American women were expected to breastfeed not only their own young, but also the infants of the white slave master and /or overseer. Sometimes, forcing the slave abandon nursing her own child. In the same instance, if a white woman had a problem breastfeeding or did not want to breastfeed, a wet nurse was secured. African American women who breasted others' infants'received better treatment than otherenslavedwomen did. This gave the perception that wet nurses were of a higher status.

However, when privileged women enlisted wet nurses, many times it resulted in the infants of wet nurses in receiving insufficient care and inferior food; thus yielding high infant mortality rates. History also tells us that thechoice to breast feed or not,a stem from various health determinates such as the mother's biology. Another one is the mother's mentalstability, family issues with production of milk, ormedication that could adversely affect/contaminate milk. 
Many times the mother's social and physical environment can shape her behavior. Thus, a major factor in the ability to breastfeed and determine which is truly better for the child. Human milk is regarded as the best source of nutrition for infants. Over recent years, it has become the preferred choice according to the American Academy of Pediatrics. Human milk provides all of the nutrients and antibodies a newborn needs to thrive and develop. Infants not fed human milk have shown higher rates of ear infections, skin rashes, intestinal infections, diarrhea, respiratory tract diseases, asthma, leukemia, and increased rate of experiencing Sudden Infant Death Syndrome (SIDS). Breastfeeding is a short-term intervention that provides long-term benefits. Infants that are formula fed have higher rates of obesity, heart disease, and Types II diabetes and I later in life.

During the Industrial Revolution, low income families used wet nurses more and more. Women began to enter the workforce, thus the inability to breastfeed. Many of the wet nurses were rural poor peasant women. With the introduction of baby formula such as Similac and Enfamil in the 1920s and 1930s, breastfeeding rates in the US drastically dropped across the board. Later, as more evidence revealed the actual health benefits of breast milk, white women resumed nursing at a higher rate.Breastfeeding rates differ substantially by race, socioeconomic level, and other demographic factors; for example, among children born during 1982-1993, non-Hispanic black children were less likely than non-Hispanic white children to be breastfed at birth - 6 months, even when comparisons were done of children in the same socioeconomic or other demographic subgroup. There is little data during this age on breastfeeding based on race, or assessing the breastfeeding education being giving by providers. The studies conducted at this time primarily focused on women as a whole. Studies also were used to assist with SIDS studies.

A comparison of breastfeeding rates and disparities were measured in the NHANES III surveysuggests that progress has been made in recent decades to increase breastfeeding initiation and decrease breastfeeding disparities between whites and blacks among economic strata. Breastfeeding initiation rates increased from $60.3 \%$ in NHANES III to $71.5 \%$ in the 2004 . The NIS demonstrated that among white children rates went from $25.5 \%$, and to $50.1 \%$ among black children. During the same period, the proportion of breastfed children who continued breastfeeding for at least 6 months increased from $44.4 \%$ to $53.9 \%$ among white children and from $33.3 \%$ to $43.2 \%$ among black children. Therefore, indicating both groups improved, but the absolute racial disparity did not diminish greatly (11.1\% in NHANES III; $10.7 \%$ in the 2004 NIS). Because of the differences that existed between NIS and NHANES III, comparisons surveys were interpreted with caution. For instance, the NHANES interview was conducted in person for children aged 12--72 months, while the NIS conducted telephone interviews and covered children aged 19--35 months. In the NHANES interview, the question was asked: "How old was [child's name] when [child's name] completely stopped breastfeeding or being fed breast milk?" In NIIS study, asked a similar question of the participants.

The CDC analyzed data from a study in 2004 called National Immunization Survey (NIS). This report indicated that $71.5 \%$ of nonHispanic white children were ever breastfed compared with $50.1 \%$ of non-Hispanic black children. Out of those ever breastfed, 53.9\% of non-Hispanic white and $43.2 \%$ of non-Hispanic black children continued breastfeeding until they were at least age 6 months. There is a large disparity between black and white children within most of the socioeconomic subgroups that were studied. Public health studies revealed that many programs lack in the promotion of breastfeeding initiation, and need to increase support of breastfeeding continuation of those subgroups with the lowest rates (i.e., black, poor and young mothers; mothers with less than a high school education; and mothers residing in rural areas).

According to the US Centers for Disease Control and Prevention's

J Pub Health Issue Pract

Volume 3. 2019. 147 most recent figures, only $62 \%$ of black babies born in the US in 2010 -started breastfeeding, compared to $79 \%$ of white babies. After six months, only $36 \%$ still breastfed, compared to $52 \%$ of white babies. The American Academy of Pediatrics recommends breastfeeding for at least the first year of life, and beyond for as long as mutually desired by mother and child. Not breastfeeding is associated with increased health risks for children, including otitis media, respiratory tract infections, diarrhea, and necrotizing enterocolitis. In addition, breastfeeding duration is inversely associated with risk for childhood overweight. Breastfeeding also is associated with health benefits for mothers, including reduced risk for ovarian cancer and premenopausal breast cancer.

African American mothers have faced a legacy of personified manipulation. In which their sexuality and reproduction were degraded just like a zoo animal on display.The abuse and demonization of their character, their bodies as women and mothers, the lack of privacyresulted in their desire to escape the judgements and manipulation that was placed on their bodies. This effect is still apparent today. African American women mentality became to reject the "animal-like" aspects of breastfeeding, according to the study done by blum in 1999. Throughout the history of the U.S,there is a constant pattern of linking the animalistic side of breastfeeding to black females, while portraying the purity of womanhood to white women. In the slave system, the southern white woman was taught to push sex out. Not only should she view her sexual organs as a shameful thing, but she should not breastfeed. She should give her children to the house slave, called the "black Mammy". The slave would have endured the white infant to suckle and nurture. According to the myth of sacred white womanhood, the white woman was above doing such "nasty "things. This type of thing is what taught the black nannie breastfeeding is improper. Black women suffered tremendously emotional, physical and psychological effects of abuse.

By moving the birthing process from midwives in the more to the hospital and physicians, ans mass producing formula society redefined the practice of breastfeeding. These changes have greatly affected breastfeeding rate. Recently, the US underwent a major change in the health policy on access/availbity to affordable health care.

Mississippi is home to a vast multitude of health issues plaguing the African Americans community. Many of which can be managed and decreased through education, which Mississippi is last when it comes to funding, and obtaining education. Which according to the textbook (Health Policy in the United States), defines health policy as the authoritative decisions made in thelegislative, executive or judicial branches of government. Usually, intended to influence or guide the actions, behavior, and decisions of citizens. Health Policies consist of one out four forms such as law, rules and regulations, operational or judicial decisions. The differences are that laws are enforced rules with an imposed penalty for failing to abide by. They are linked to all parties and citizens. Whereas rule and regulations belong to an agency and the future, effect is to implement, interpret, or prescribe law. Judicial is considered a court ruling; operation dictates the laws, rules on maneuvers.

Less than 4 percent of U.S. hospitals offer the full range of support services that new mothers need to master breast-feeding, a new government report shows. This is an important issue because breastfeeding protects against childhood obesity and offers other health benefits to children, according to the Vital Signs report released online Tuesday by the U.S. Centers for Disease Control and Prevention.

Researchers analyzed data from CDC's national survey of Maternity Practices in Infant Nutrition and Care, and found that only 14 percent of hospitals have a written breast-feeding policy. In nearly 80 percent of hospitals, healthy breast-feeding infants are given formula when it is not medically necessary. This practice makes it much more difficult for new mothers and infants to learn how to breast-feed and

JPHIP, an open access journal ISSN- 2581-7264 
to continue breast-feeding at home.

The findings in this report indicate substantial racial and economic disparities in rates of breastfeeding initiation and breastfeeding continuation to at least age 6 months. The findings also demonstrate that race is associated with breastfeeding status independent of socioeconomic and other demographic factors, but also that socioeconomic and other factors are associated with breastfeeding independent of race. Within each income group, the proportion of black children who were ever breastfed was 10 to 17 percentage points lower than that of white children; within each race, the proportion of children ever breastfed was 23 to 26 percentage points higher among those in the highest income group compared with the lowest. Racial differences in breastfeeding continuation rates to 6 months were generally smaller than differences observed in breastfeeding initiation. Among the other findings were:

- Only one-third of hospitals practice "rooming in," in which mothers stay with their newborns 24 hours a day. This helps mothers and newborns learn to breast-feed by giving them frequent opportunities to breast-feed.

- Nearly, 75 percent of hospitals do not provide necessary breastfeeding support to mothers and babies when they leave the hospital. Required support includes a follow-up visit, a phone call from hospital staff and referrals to lactation consultants and breast-feeding support systems in the community.

Low rates of breast-feeding in the United States result in $\$ 2.2$ billion in additional medical costs per year. Babies who are fed formula and stop breast-feeding early have higher rates of obesity, diabetes, respiratory and ear infections. They also require more doctor visits, hospitalizations and prescriptions, according to U.S. Centers for Disease Control and Prevention, news release.

Parents can save thousands of dollars during their baby's first year of life by breastfeeding, Along these same lines, parents will save money on health care bills since breastfed infants get sick less often that their formula fed counterparts and are also hospitalized less often.

Breastfeeding is good for the economy. A 2010 study analyzed the costs of treating ten common childhood illnesses, including hospitalization, and the amount of money that could be saved if $90 \%$ of women breastfed their babies exclusively for the first six months of life. The study estimated that the U.S. could save 13 billion dollars annually if that breastfeeding goal were met.

Breastfeeding is good for the environment. In addition to these savings, breastfeeding helps our country's bottom line. From the Surgeon General's Call to Action to Support Breastfeeding, "Breastfeeding also confers global environmental benefits; human milk is a natural, renewable food that acts as a complete source of babies' nutrition for about the first six months of life. Furthermore, there are no packages involved, as opposed to infant formulas and other substitutes for human milk that require packaging that ultimately may be deposited in landfills.

Early exposures during the critical periods of childhood influence adult health. Identifying the social factors that influence maternal breastfeeding behaviors will move program and policy to take specific actions: Incorporation of life course into standard data reportingand public health approaches. Using newly available PRAMS data to explore self-reportedmaternal barriers to breastfeeding initiation and duration. More display of breastfeeding materials that positively promote and protect breastfeeding mothers.

Enlightening the public on the positive effect, it has on the environment. For every one million formula-fed babies, 150 million containers of formula are consumed; while some those containers could be recycled, many end up in landfills. It generally requires no containers, no paper, no fuel to prepare, and no transportation to deliver, and it reduces the carbon footprint by saving precious global resources and energy.

Although racial and economic disparities in breastfeeding initiation rates appear to have decreased in recent decades, they have not been eliminated. Barriers to breastfeeding initiation and continuation include lack of social support, lack of proper guidance from healthcare providers, lack of adequate or timely postpartum follow-up care, and disruptive hospital maternity-care practices such as delays in breastfeeding initiation due to the use of pacifiers by newborns, and hospital promotion of formula through the provision of free formula in hospital discharge packs. Increasing rates of breastfeeding is a crucial strategy for improving children's health, reducing childhood overweight, and reducing health-care costs. For example, increasing the proportion of children breastfed in the early postpartum period from $64 \%$ in 2000 to the Healthy People 2010 goal of $75 \%$ would save an estimated $\$ 3.6$ billion in health-care costs annually.

In order to engage, more African American mothers we must approach breastfeeding by recognizing social, cultural and political; as well as the historical contexts. Our policy makers and other groups of interest need realize that there is a decreasing breastfeeding disparity. The true way to battle through is by improving breastfeeding initiation and duration. This should be done by allocating breastfeeding interventions for racial, ethnic, and socioeconomic variations in attitudes towards breastfeeding and perceived barriers to breastfeeding.

More Public health measures to promote breastfeeding should continue and should target groups with the lowest initiation rates, such as black mothers in rural areas and/or mothers under 20 years old, mothers who have not completed high school, and those who participants in the WIC (Women, Infant, Children) program. There is a critical need for more Public health programs that will increase protection and support of breastfeeding continuation among the groups. The CDC Guide to Breastfeeding Interventions main function is introducing interventions aimed at promoting and supporting breastfeeding.

Conflict of interest: The authors declare no conflict of interest.

\section{Reference}

1. Exclusive breastfeeding (2016).

2. Longest B. Jr. (2009) Health Policymaking in the United States (5th ed.). Health Administration.

3. The Surgeon General's Call to Action to Support Breastfeeding. (2015, June 17).

4. Health Disparities (2016) Background and Research Summary. (n.d.).

5. Shely K, Li R, Benton-Davis S, Grummer-Strawn L (2005) He CDC Guide to Breastfeeding Interventions. Atlanta: U.S. Department of Health and Human Services, Centers for Disease Control and Prevention, . The Center for Disease Control.

6. Political Circumstance (2004) Found in Many U.S. Hospitals Lacking Support for Breast-Feeding. August 2. HealthNews Daily. 